\title{
The challenge of reducing medication errors in neonatal intensive care units
}

Errors have always been present in medicine, and even though many resulted in damage, including death, nobody talked about them since the prevalent culture was that of concealment and of punishment imposed on those responsible for them. There were no prevention measures in place and no possibility of learning from mistakes, both critical steps in error prevention. The history changed when an investigation was carried out. It was a historic landmark because it "opened the doors" for what we now call patient safety. That study, led by Doctor Leape from Harvard University, ${ }^{1}$ proved to the world that approximately 100,000 hospitalized patients died every year in the USA due to medical care errors (more than twice that number die at present). Then, in our setting, we became more aware of the need to admit that everybody makes mistakes in medical practice. This set about the difficult steps towards culture change, which is still a huge challenge today. Such change enabled approaching relevant aspects, including error analysis, prevention measures, learning from other risky professions, designing protection barriers so that errors do not reach patients, improving communication among hospital team members, and turning patients and parents into our partners. This was the path to our present day; major advances have been made but there is also the certainty that we still have a long road ahead and that we should strongly advocate for medical facilities to provide reliable safety in their patients' best interest.

At least in Argentina, there are still several aspects that promote a continuing inadequate safety culture. Medical professionals, as well as nurses and everyone else involved in health care, do not bear in mind that errors are part of the human condition and will inevitably occur. In addition, we have not been properly trained on how to face errors, neither during our university education nor during our early medical training; on the contrary, there is a false assumption still in place that states that no mistakes are made in the medical profession because there is a prevalent though inadequate concept regarding our activity: infallibility.

In relation to neonatal care, everybody agrees that there is a greater risk of making mistakes in this discipline than in others, especially in neonatal intensive care units (NICUs). This is predominantly due to an impaired hospital system and the high complexity of care, mostly motivated by the increasing survival rate of extremely preterm infants and newborn infants with severe malformations, both highly vulnerable populations. Even with the differences observed in the prevalence rates among units, it is usually considered that medication errors are the most common type observed in the NICU, 2,3 together with nosocomial infections. In the USA, medication errors account for approximately 700000 visits to the emergency room and more than 100000 hospitalizations every year. Adverse drug reactions (ADRs) occur in 5\% of hospitalized patients, and are one of the most common errors detected in hospitals.

There is a great variation in the medication error rate in the NICU. It was observed to be $22 \%$ in a study conducted in the Hospital Italiano de Buenos Aires. ${ }^{2}$ However, in the US Vermont Oxford Network it was over $40 \%{ }^{3}$ An extensive study on errors made in children, in which $70 \%$ occurred in newborn infants, $0.6 \%$ had an adverse event, $78 \%$ were preventable errors, and the most common cause $(21 \%)$ was a medication error. ${ }^{4}$ In a recent study, $49 \%$ of medication errors in the NICU decreased to $31 \%$ after boosting the safety culture. ${ }^{5}$

The adverse event rate in newborn infants is three times higher than in adults, and their mortality rate is also higher. This is even more relevant when considering the undeniable results of several studies that indicate that ADRs may cause long-term neurological development disorders. This is even more concerning in the case of extremely preterm infants given that certain drugs will increase their already elevated risk of potentially irreparable consequences. ${ }^{6}$

It is worth noting that these errors are mostly related to medication process failures, an excessive reliability on memory, and lack of attention during prescription and administration. The high rate of medication errors in the NICU is related to several risk factors. The main risk factors include: doses are estimated on a weight basis and most need to be diluted, almost all medications are administered intravenously, and more than 80\% are not approved for use in newborn infants and doses are extrapolated from those indicated for children and adults. 
Drug dilution is very prone to serious mistakes, which tend to occur when it has to be repeated more than once, which is not uncommon, mainly in small preterm infants. The most common error occurs when a ten-fold (or even higher) dose is administered, which will frequently result in death.

To assess why these medication errors occur, we could use the model proposed by Reason in his book Human Error, ${ }^{7}$ which has been used to assess the causes of disasters that took place in highly risky industries, and allows us to examine errors from three different perspectives: individuals, immediate surrounding, and organizational culture. If this model is extrapolated to medication errors, we may assess them from the three perspectives. First of all, the responsibility and ability of the prescribing physician and the administering nurse. Residents, who are the ones that prescribe medications, are not usually trained on how to do it, and there are no guidelines in place that could facilitate an adequate implementation. Two main assumptions should be emphasized to prevent errors: neither physicians nor nurses should rely on their memory, because it is fallible, and both drug prescription and preparation should be reviewed by a third party to ensure they are correct. This intervention demonstrated its effectiveness in multiple risky activities but unfortunately it is rarely implemented in medical practice. The second aspect is related to an inadequate setting at the time of prescription and administration. Multiple interruptions and disturbing noises are common in the NICU and affect the different tasks that take place there. A study that assessed 784 medication errors observed that $90 \%$ were motivated by distractions in the medication process. ${ }^{8}$ The third aspect is the importance given to safety culture in medical institutions. In my opinion, this is the most important element because making a change in traditional culture is really difficult, and while this lasts, no achievements will be made in relation to the aspects described here and there will be no motivation to create an environment where safety and patient injury prevention are the top priorities.

In addition, I would like to emphasize that, apart from the measures discussed here, it is fundamental to carry out other actions in order to reduce errors and prevent adverse events. These include, mainly, adequately identifying patients, carrying out root cause analyses of errors to learn from them and prevent their repetition, implementing prevention strategies and barriers, and also asking parents to become our partners so that they can help us in the difficult challenge that means preventing errors in their children.

Only in this way we will be able to reach a higher safety level in neonatal care units and thus reduce the rate of adverse events in newborn infants, which is undoubtedly an inherent ethical mandate.

José M. Ceriani Cernadas Editor

http:/ /dx.doi.org/10.5546/aap.2016.eng.394

\section{REFERENCES}

1. Brennan TA, Leape LL, Laird NM, Hebert L, et al. Incidence of adverse events and negligence in hospitalized patients. Results of the Harvard Medical Practice Study I. N Engl J Med 1991;324(6):370-6.

2. OteroP,Leyton A,MarianiG,CerianiCernadasJM.Medication errors in pediatric inpatients: prevalence and results of a prevention program. Pediatrics 2008;122(3):e737-43.

3. Suresh G, Horbart JD, Plsek P, Gray J, et al. Voluntary anonymous reporting of medical errors for neonatal intensive care. Pediatrics 2004;113(6):1609-18.

4. Thomas EJ, Studdert DM, Burstin HR, Orav EJ, et al. Incidence and types of adverse events and negligent care in Utah and Colorado. Med Care 2000;38(3):261-71.

5. Chedoe I, Molendijk H, Hospes W, Van den Heuvel ER, et al. The effect of a multifaceted educational intervention on medication preparation and administration errors in neonatal intensive care. Arch Dis Child Fetal Neonatal Ed 2012;97(6):F449-55.

6. Yeh TF, Lin YJ, Lin HC, Huang CC, et al. Outcomes at school age after postnatal dexamethasone therapy for lung disease of prematurity. N Engl J Med 2004;350(13):1304-13.

7. Reason J. Human error: models and management. BMJ 2000;320(7237):768-70.

8. Marino BL, Reinhardt K, Eichelberger WJ, Steingard R. Prevalence of errors in a pediatric hospital medication system: implications for error proofing. Outcomes Manag Nurs Pract 2000;4(3):129-35. 